\title{
Comparison of the epidural block and posterior lumbar plexus block for intra- and postoperative analgesia in total hip arthroplasty
}

\author{
Bianca BASGAN ${ }^{1}$, Niculae IORDACHE ${ }^{2,3}$, Dragos COCOS $^{4}$, Iuliana Raluca GHEORGHE 5 \\ ${ }^{1}$ Department of Anesthesiology and Intensive Care Medicine, "Saint Elisabeth Hospital" Gütersloh, \\ Germany \\ 2Department of Surgery, "Saint John" Emergency Clinical Hospital Bucharest, Romania \\ ${ }^{3}$ Clinical Department no. 10, "Carol Davila" University of Medicine and Pharmacy Bucharest, Romania \\ ${ }^{4}$ Department of Anesthesiology and Intensive Care Medicine, "Marienhospital" Oelde, Germany \\ ${ }^{5}$ Department Health Care Marketing and Medical Devices, "Carol Davila" University of Medicine and \\ Pharmacy Bucharest, Romania
}

\begin{abstract}
Objectives. Evaluation of management of intra- and postoperative analgesia in patients who benefited from total hip arthroplasty in the surgical treatment of coxarthrosis. The achievement of this goal could reside in the comparison of two distinct methods of anaesthesiology: epidural analgesia and continuous lumbar plexus block.

Materials and methods. The current prospective study, conducted between January 2014 and January 2016, targets a total of 48 patients who underwent total hip replacement surgery. There was a split in two groups: 23 patients benefited from epidural analgesia and 25 patients were selected for lumbar plexus block, depending on the procedure chosen by the anaesthetist. Both groups received the same concentration of initial dose of $0.75 \%$ Ropivacaine, the first group initially receiving $3 \mathrm{ml}$ (test dose), then another $6 \mathrm{ml}$, and the second initially $5 \mathrm{ml}$ (test dose), then $15 \mathrm{ml}$. Through catheters were injected continuously $8 \mathrm{ml} / \mathrm{h}$ of $0.2 \%$ Ropivacaine for the first group and $10 \mathrm{ml} / \mathrm{h}$ of Ropivacaine $0.375 \%$, these doses being adjusted to Pain Visits, performed twice a day. The purpose of these visits was to monitor both the evolution of implanted catheters and the completion of mandatory protocols (these protocols monitor sensitivity and motor activity of limbs, initiation of mobilization, opioid supplementation to ongoing therapy, and patients' satisfaction with therapy, at catheter removal).

Results. Patients in the first group had the highest satisfaction rate of $30.4 \%$, compared to patients in the second group, who scored 52\% for the 15 (maximum) level of satisfaction. In the first group of patients, $52.2 \%$ did not require additional opioids for ongoing therapy (in postoperative units or in the department), while for patients in the second group the percentage was $60 \%$. In terms of complications, the differences are notable and have an important statistical value: the first category of patients, $52.17 \%(n=12)$, the second category, $12 \%(n=3)$. Day zero (surgery day) is not marked by significant differences between the two categories of people in treatment, in terms of pain intensity. At rest, $78.2 \%$ of the first category patients and $84 \%$ of the second category patients had a NRS of less than or equal to 4 , while in the state of mobilization, the percentages were $78.2 \%$ and $80 \%$ respectively. The initiation of mobilization is an important parameter, which also
\end{abstract}


presents variations between the two groups of patients: $73.9 \%$ of them initiated the mobilization on the first postoperative day (group I) and 88\% initiated the mobilization on the first postoperative day (group II).

Conclusions. Both methods of regional anaesthesia determine optimal analgesia for patients operated by THA with an onset of ideal mobilization from the first postoperative day, but the continuous lumbar plexus block produces better mobility in the second postoperative day and has a lower complications rate.

Keywords: continuous lumbar plexus block, epidural analgesia, psoas muscles, total hip arthroplasty

\author{
Abbreviations \\ ASA - American Society of Anethesiologists \\ GCS - Glasgow Coma Scale \\ NRS - Numeric rate scale
}

\author{
PDK - Peridural Catheter \\ PONV - Postoperative Nausea and Vomiting \\ THA - Total Hip Arthroplasty
}

\section{INTRODUCTION}

The management of pain in patients undergoing THA has long been debated over time, constituting a permanent challenge for anaesthetists, as treating patients with preoperative chronic pain or elderly patients claiming postoperative acute pain has never been simple. As THA surgery currently has a very high incidence in evolving countries, reaching four operations per day in a medium-sized hospital or more in some orthopaedic departments, it is obvious that the implementation of appropriate pain therapy in the coxarthrosis treatment has become an imperative.

There is a disagreement about the standardization of the therapy in the literature, and today there are a number of potential solutions for developing an ideal technique for intra- and postoperative analgesia, especially the first 24 hours after the surgical cure. Among these solutions we mention: pure drug therapy, patientcontrolled analgesia (i.v.) with opioids, epidural analgesia with continuous injection of local anaesthetic with or without opioids, femoral nerve block and psoas compartment block, the latter eventually combined with sciatic nerve block $(1,6,7,11)$.

Ideally, the pain therapy should use methods with minimal side effects (nausea, vomiting, hypotension, collapse, urinary retention, fatigue), but at the same time with great results for their quality of life, with primordial initiation of physiotherapy from the first postoperative day to recover as quickly as possible and to avoid other complications arising from a long stay in the bed: various postoperative infections, muscle atrophy due to prolonged rest, contractions of the back or neck muscles, articular thrombosis due to immobility, deep venous thrombosis etc.
The rapid onset of physiotherapy exercises, the minimal doses of opioids administered to recovery patients, or a visible improvement in general condition and patient mood are just some of the immediate benefits that the regional anaesthesia technique, understood as a coherent, patient-focused method of postoperative analgesia, most often generates.

If the technique of epidural catheter implantation is particularly preferred by anaesthetists, lumbar plexus block is unfortunately not a common method in all major hospital centres, as it involves qualified staff but also the allocation of additional material resources. These can currently be seen as major impediments to the implementation as a routine medical practice of this method in Eastern European hospitals.

\section{METHODS}

The imperative of finding an effective method for pain therapy is the fundament of this prospective study, which proposes a series of bold goals, including: comparing the patients' satisfaction for catheter extraction expressed by them on a scale from 0 to 15 ; marking the moment of onset of medical gymnastics, establishing the need for intra- and postoperative opioids; assessing the analgesic effect of both catheters, which have the role of minimizing opioid use; calculating the percentage of patients with a NRS $\leq 4$ pain level on the surgery day and on the first postoperative day, both at rest and at mobilization; assessing complications that may occur throughout pain therapy stages.

The patients participating in this study, diagnosed with coxarthrosis, were between 18 and 80 years of age and the ASA classification between I and III. 
Amongst the exclusion criteria we may include: drug allergies to local anaesthetics used in the study, morbid obesity, lumbar infection of the area to be punctured, failure of planned catheter binding.

Preoperatively, patients received the following drugs: Midazolam 3.75/7.5 mg p.o. age-appropriate dose and taking into account the existence of chronic conditions, Oxycodone $10 \mathrm{mg}$ p.o. and Etoricoxib $90 \mathrm{mg}$ p.o. After the pain catheter binding, a $0.75 \% 3 \mathrm{ml}$ Ropivacaine dose for the epidural catheter (group I patients) and $5 \mathrm{ml}$ for the lumbar plexus block catheter (group Il patients) were administered. After 5 minutes the therapeutic dose, consisting of $6 \mathrm{ml}$ for the first group and $15 \mathrm{ml}$ for the second, followed by the general anaesthesia. During these procedures, as well as during or postoperative surgery, patients are continuously monitored hemodynamically.

In the recovery room, patients stayed several hours while receiving Piritramid i.v. for a pain level greater than 4 on the NRS scale $(0-$ no pain, 10 - severe pain). From here, the transfer was made to the surgical ward, where the pain catheter was connected to an infuser. Patients received $0.2 \%$ Ropivacaine at a dose of $6 \mathrm{ml} / \mathrm{h}$ (first group) and $0.375 \%$ Ropivacaine at a dose of $10 \mathrm{ml} / \mathrm{h}$ (second group) as well as Metamizol $5 \mathrm{~g}$ daily (both groups) as adjuvant therapy. None of the patients had a known allergy or had any allergic reaction to Metamizol, so all patients received these drops. Opioids (Oxycodon $5 \mathrm{mg}$ Akut p.o.) could be given to the section at any time if the patient's condition required this (if NRS - greater than or equal to 4). At Pain Unit Visits, which is done twice a day, an anaesthetist with a pain nurse visits the patients and fills in the protocol for these visits, including: the degree of pain at rest and at mobilization, the degree of sensitivity and/or motricity of both limbs, controlling the place where the catheter is placed, and changing the bandage as needed, assessing complications (nausea, vomiting, collapse, hypotension). Each of the two catheters remain attached for at least 48 hours, within Pain Unit Visits, reducing the dose according to patient's needs. Subsequently, the patient's reaction to discontinuation of Ropivacaine over a 12-hour period is tested from the third or, if appropriate, the fourth day. If the pain remains between 0 and 4 NRS, both at rest and at mobilization, and the patient is satisfied, then the catheter can be removed, otherwise drug administration continues at a reduced dose.
Epidural analgesia is performed at lumbar level L3-L4 or L4-L5, between dura mater and yellow ligament, and epidural space detection is performed by Dolgiotti technique (loss of injection resistance).

Localization of the lumbar plexus is accomplished with peripheral nerve stimulator. The lower limb is, through the ilioinguinal genitofemural, ileohipogastric and lateral femoral cutaneous (collateral branches) nerves or obturator nerve, accessory and femoral obturator (terminal branches) nerves, sensitively and motrically serviced by the lumbar plexus, anatomically positioned between the muscular plans of the large psoas muscle and made up by combining the anterior branches of the T12-L4 spinal nerves.

Puncture is done by drawing a sign at a distance of $3 \mathrm{~cm}$ below L4, from which it is measured at an angle of 90 degrees another $5 \mathrm{~cm}$ laterally, after the patient having previously been placed in lateral decubitus or sitting.

In terms of medical statistics, this was achieved through SPSS programe, version 20.0. Comparative viable results were obtained between the two groups of patients, those with a statistical value being considered as having a $p$ value $<0.05$ (Chi-square Test).

\section{RESULTS}

This study targeted a number of 48 patients and tracked a series of important data for the comparative assessment of the two catheterization techniques, but only 11 parameters were statistically analysed:

Patient satisfaction from 0 (extremely dissatisfied) to 15 (very satisfied); only patients with grade 15 were registered here, with $30.4 \%$ $(n=7)$ being delimited in the first group and $52 \%(n=13)$ in the second group, a clear difference but not statistically significant ( $p$ value $=$ 0.13)

The onset of mobilization occurred in most patients on the first postoperative day, but there was no significant difference between the two groups (73.9\% vs. $88 \%)$.

Also in terms of mobilization: going to the toilet with support is a parameter with statistical significance ( $p$ value $<0.05$, more precisely 0.01 ), the difference bringing advantage to the group that received lumbar plexus block. Patients in this group (group II) managed to go to the toilet with $84 \%$, compared with the first group (only 52.2\%). 
Intraoperative opioid graduation was divided into 3 subgroups: $0.1 \mathrm{mg}$ Fentanyl, $0.15 \mathrm{mg}$ Fentanyl and $0.2 \mathrm{mg}$ Fentanyl, these being the most frequent doses administered to both groups. Based on these premises, the following subgroups are registered: $26.1 \%$ epidural group and $8 \%$ psoas group, $26.1 \%$ and $40 \%$ second subgroup, and in the third subgroup $21,7 \%$ vs. $28 \%$, all of which have no statistical value.

Additional opioid administration (in the postoperative unit or in the surgical ward) brought again some differences, but with no statistical significance, this being $47.8 \%$ of the patients in the epidural group, respectively $40 \%$ of the patients in the psoas group have required an additional opioid for ongoing therapy.

Intensity of pain at day 0 (surgery day) at rest showed two encouraging percentages for both groups of patients: $78.3 \%$ of those with the epidural catheter and $88 \%$ of those with continuous lumbar plexus block have had a NRS $\leq 4$ at Pain Visits performed in the evening, after the surgery.

Intensity of pain in the first postoperative day at rest demonstrates once again that patients in both groups do not claim severe pain with NRS $\leq 4$ due to pain catheters: $87 \%$ of the epidural catheterization patients and $80 \%$ of the patients those belonging to the psoas group.

Intensity of pain in the first postoperative day at mobilization, NRS $\leq 4$, shows some percentages lower than at rest, but still consistent: $65.2 \%$ for the epidural group vs. $68 \%$ for the psoas group.

Postoperative complications include subcutaneous hematoma, lower limb sensitivity disorders up to complete motor block, collapse, hypotension. In the postoperative complications, a parameter with a significant statistical value ( $p$ value $<0.05$, namely 0.03 ) was again in favour of the second group, the percentages being $52.2 \%$ for those with an epidural catheter, respectively $12 \%$ for those with lumbar percutaneous catheter.

Total catheter duration: for the first group a mean maintenance time of 4.34 days was calculated and for the second group of 4.8 days.

Intraoperative hypotension mean (after catheterization) did not show a statistically significant differentiation, this being a value of $59.44 \mathrm{mmHg}$ for group I and $63.57 \mathrm{mmHg}$ for group II.

In the figures below are shown the two parameters with significant statistical value, $p$ value $<0.05$ : advanced mobilization in the second postoperative day (going to the toilet with support) and complications in each group after the surgery. Both parameters advocate continuous lumbar plexus block, on the one hand patients in this group succeded in $84 \%(n=21)$ to get up from bed and even go to the toilet with the physiotherapist's help, and on the other hand the number of complications from the epidural catheter, $52.17 \%(n=12)$, represents a significant disadvantage compared with those in the psoas group where only 3 patients suffered from complications (Fig. 1, Fig. 2).

\section{DISCUSSIONS}

All the results described above are relevant and reliable at the same time, providing useful information with an avant-garde trend for future approach to intra- and postoperative pain management, following THA interventions.

The start of mobilization is a relevant parameter, and can be regarded as the result of a perfect collaboration between anaesthetist, physiotherapist and patient. Without the correct input from one of the parties, the results presented previously in our study would not have been possible. The high percentages of both groups initiating mobilization from first postoperative day ( $73.9 \%$ vs. $88 \%)$ bring an optimistic trend for those anaesthetist colleagues who strongly support that regional anaesthesia does not interfere with the performance of medical gymnastics and make constant efforts to persuade some orthopaedists, more reluctant to the novelty, of this state of facts. Even though the difference between the two groups is not statistically relevant, the psoas group's higher percentage of early mobilization is nevertheless seen as a real success for this regional method.

Remaining in the same mobilization register, we can announce, from a statistical perspective, that mobilization in the second postoperative day, namely going to the toilet with support, recorded a $p$ value $=0.01$, as a significant difference between the two patient groups. Thus, we can reveal a percentage of $84 \%$ of the patients with lumbar plexus block that were mobilized, compared with only $52.2 \%$ of the epidural group.

Generally speaking, mobilization is a decisive step in recovering patients after any surgery. Any method that brings benefits in this regard should be debated interdisciplinary and then imposed as a preoperative standard. 


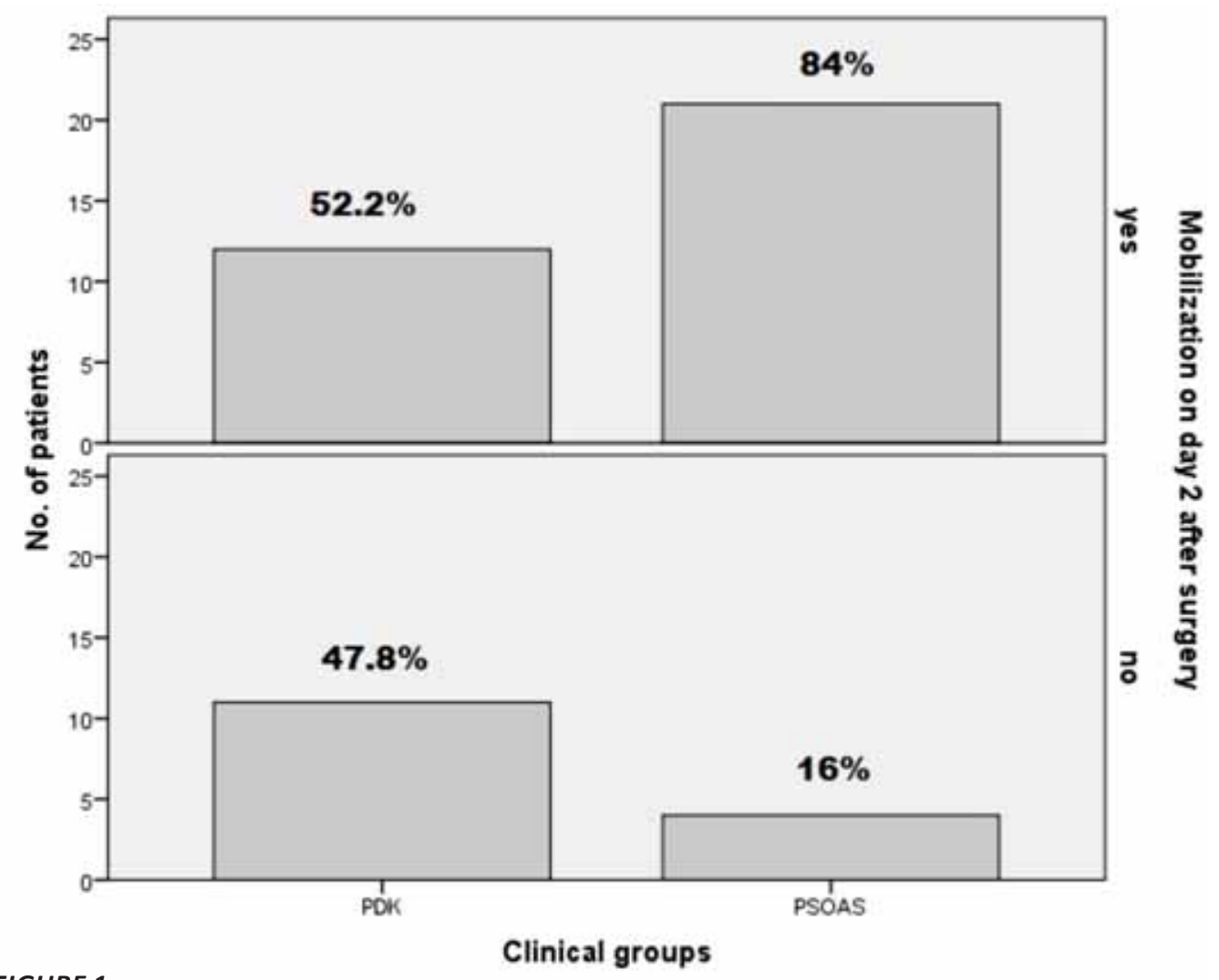

FIGURE 1

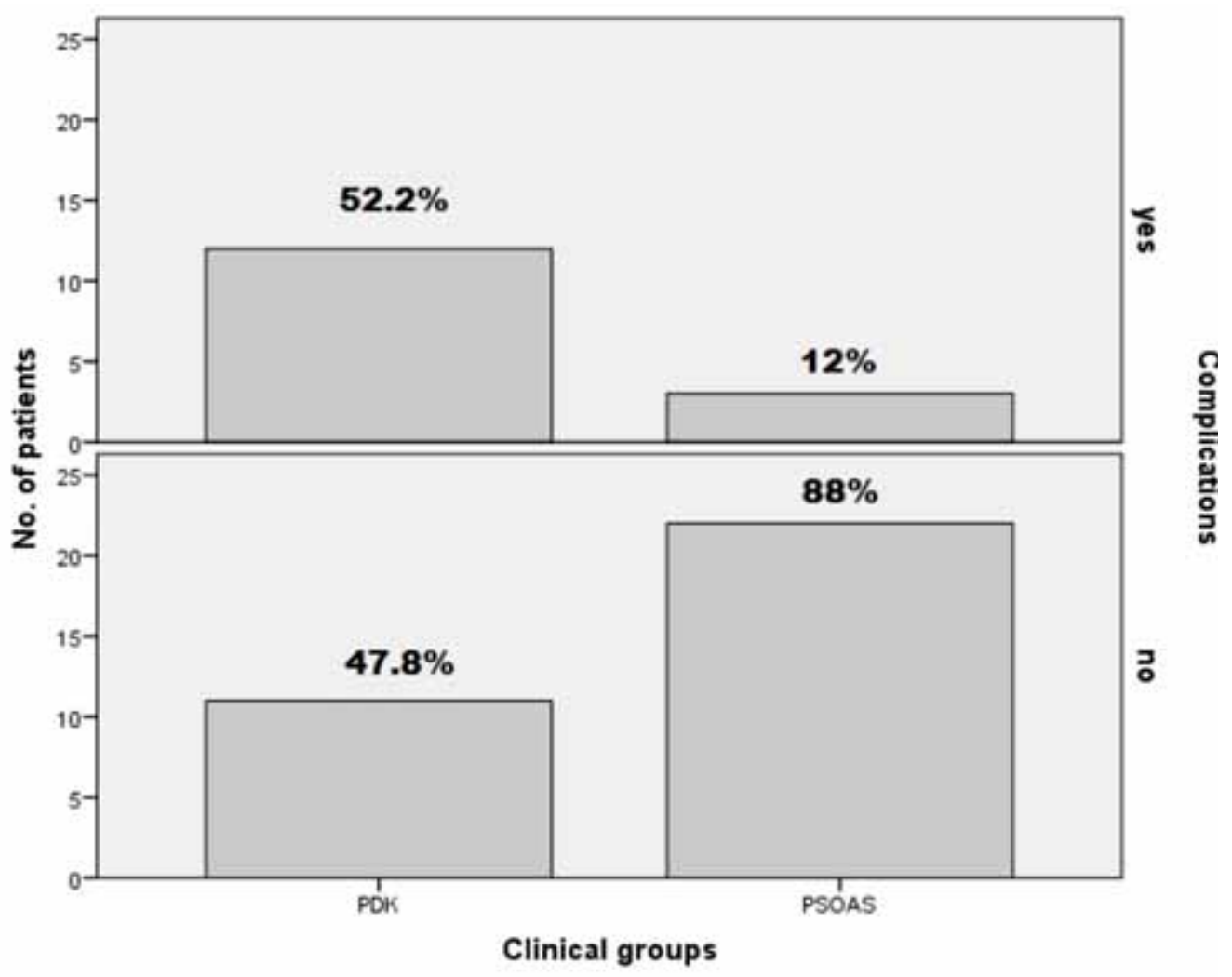

FIGURE 2 
Interestingly enough is the evaluation of the ancillary opioid needs in both the Recovery Room and the ward. Here, the balance leaned again in favour of the psoas group, with $60 \%$ of patients using baseline therapy alone, with no opioid surplus, compared with $52.2 \%$ in the epidural group. Trying to minimize opioid use is a vital desideratum that should be made aware of all colleagues who are using these drugs too easily.

The intensity of pain monitored on the surgery day (day 0 ) and on the first postoperative day at rest and at mobilization reveals adequate analgesia with comparable effects for both anaesthetic procedures. On the first postoperative day, a total of over $80 \%$ of patients reported a NRS $\leq 4$ at rest, and over $60 \%$ of the patients had a similar degree of pain at mobilization.

In the "Complications" chapter there is again a significant difference between the two groups, $p$ value $=0.03$, the epidural technique generating more undesirable complications. It should be noted that from our study, nausea and vomiting were excluded as complications because they have no direct connection with regional techniques, but rather with general anaesthesia.

We underline at this point that no dissemination of local anaesthetic into the epidural space and no bilateral analgesia were observed at the patients with lumbar plexus block.

From hemodynamic point of view, both groups remained intraoperatively stable, the hypotension average being very close to the value of $60 \mathrm{mmHg}$, a common value in clinical practice in the clinic where the current study was conducted, with only a small difference in favour of the psoas group.

\section{CONCLUSIONS}

This study succeeds in clarifying some very important aspects of the comparative assessment of the epidural catheter and the continuous lumbar plexus block in the THA surgery, the latter presenting a range of certain advantages: early mobilization from the first day after the surgery; frequency of second day mobilization, expressed by the higher number of patients who performed medical gymnastics; a higher percentage of patients who did not receive opioids in the Recovery Room or the surgical ward during the catheter maintenance period; fewer postoperative complications of the catheterization method and drug delivery via catheter.

Technically speaking, continuous lumbar plexus block appears to be one step ahead of epidural analgesia in terms of mobilization and complications. All these favourable premises being drawn, we can say with optimism that the parameters of this study will certainly be used as a consistent basis in the elaboration of other multicentre studies.

Conflict of interest: none declared Financial support: none declared

\section{REFERENCES}

1. A. Chudinov, H. Berkenstadt, M. Salai, A. Cahana and A. Perel, „Continuous psoas compartment block for anesthesia and perioperative analgesia in patients with hip fractures", Regional Anesthesia and Pain Medicine, 1999, 24 (6), 563-568

2. Robert D. Stevens, Elisabeth Van Gessel, $\dagger$ Nicolas Flory, $\ddagger$ Roxane Fournier, $\ddagger$ Zdravko Garnulin, “'Lumbar Plexus Block reduces Pain and Blood Loss associated with Total Hip Arthroplasty ", Anesthesiology, 2000, 93 (1), 115-121

3. X. Capdevila, P. Macaire, C. Dadure, 0 . Choquet, P. Biboulet, Y. Ryckwaert et al., „Continuous Psoas Compartment Block for postoperative Analgesia after Total Hip Arthroplasty: new Landmarks, technical Guidelines and clinical Evaluation", Anesthesia and Analgesia, 2002, 94(6), 1606-1613

4. J.E. Chelly, A. Casati, T. AI-Samsam, K-Coupe, A. Criswell, J. Tucker,
„Continuous lumbar Plexus Block for acute postoperative Pain Management after open Reduction and internal Fixation of actebular Fractures", Journal of Orthopaedic Trauma, 2003, 17(5), 362-367

5. G. Türker, N. Uckunkaya, B. Yavascaoglu, A. Yilmazlar, S. Ozcelik, "Comparison of the catheter-thechnique psoas compartment block and the peridural block for analgesia in partial hip replacement surgery", Acta Anaesthesiologica Scandinavica, 2003, $47(1), 30-36$

6. P. Biboulet, D. Moran, P. Aubas, S. Bringuer - Branchereau and X. Capdevila, „Postoperative Analgesia after Total Hip Arthroplasty: Comparison of intravenous patient-controlled Analgesia with Morphine and single Injection of femoral Nerve or Psoas Compartment Block: a prospective, randomized, double-blind Study", Regional Anesthesia and Pain Medicine, 2004, 29(2), 102-109
7. S. Ganidagli, M. Cengiz, Z. Baysal, L. Baktiroglu and S. Sarban, „The Comparison of two lower Extremity Block Techniques combined with sciatic Block: 3-in-1 femoral Block vs. Psoas Compartment Block", International Journal of Clinical Practice, 2005, 59(7), 771-776

8. Y. Asao, T. Higuchi, N. Tsubaki, Y. Shimoda, "Combined paravertebral lumbar Plexus and parasacral sciatic Nerve Block for Reduction of Hip Fracture in four Patients with severe Heart Failure", The Japanese Journal of Anesthesiology, 2005, 54(6), 648-652

9. S. Mannion, S. O'Callaghan, M. Walsh, D. B. Murphy and G.D. Shorten, „In with the new, out with the old? Comparison of two Approaches for Psoas Compartment Block", Anesthesia and Analgesia, 2005, 101(1), 259-264

10. X. Capdevila, C. Coimbra, O. Choquet, „Approaches to the lumbar Plexus: Succes, 
Risks, and Outcome", Regional Anesthesia and Pain Medicine, 2005, 30(2), 150-162

11. Gisela Meyer, Johannes Büttner, „Regionalanästhesie: Kompendium der peripheren Blockaden", 2006

12. Z.I. Siddiqui, M.S. Cepeda, W.Denman, R. Schumann and D.B. Carr, "Continuous lumbar Plexus Block provides improved Analgesia with fewer side effects compared with systemic Opioids after Hip Arthroplasty: a randomized controlled Trial", Regional Anesthesia and Pain Medicine, 2007, 32(5), 393-398

13. S. Mannion, „Psoas Compartment Block”, Continuing Education in Anesthesia, Critical Care and Pain, 2007, 7(5), 162-166

14. M.A. de Leeuw, J. A. Dertinger, L. Hulshoff et al., „The Efficacy of Levobupivacaine, Ropivacaine and Bupivacaine for combined Psoas Compartment - sciatic Nerve Block in
Patients undergoing Total Hip Arthroplasty", Pain Practice, 2008, 8(4), 241-247

15. J.C. Gadsden, D.M. Lindenmuth, A. Hadzic, D. Xu, L. Somasundarum and K. A. Flisinski, ,Lumbar Plexus Block using high-pressure Injection leads to contralateral and epidural Spread", Anesthesiology, 2008, 109(4), 683-688

16. L.T. Duarte, F.C. Paes, C. Fernandes Mdo, R. A. Saraiva, „Posterior Lumbar Plexus Block in postoperative Analgesia for Total Hip Arthroplasty: a comparative Study between $0,5 \%$ Bupivacaine with Epinephrine and $0,5 \%$ Ropivacaine", Brazilian Journal of Anesthesiology, 2009, 59(3), 273-285

17. L.T. Duarte, P.S. Beraldo, R.A. Saraiva, „Effects of epidural analgesia and continuous lumbar plexus block on functional rehabilitation after total hip arthroplasty", Brazilian Journal of Anesthesiology, 2009, 59(5), 531-544
18. L.T. Duarte, P.S. Beraldo, R.A. Saraiva, „Epidural Lumbar Block or Lumbar Plexus Block combined with general Anesthesia: Efficacy and hemodynamic Effects on Total Hip Arthroplasty", Brazilian Journal of Anesthesiology, 2009, 59(6), 649-664

19. M.A. de Leeuw, W. W. A. Zuurmond, R.S. G.M. Perez, „The Psoas Compartment Block for Hip Surgery: The Past, Present and Future", Anesthesiology Research and Practice, 2011, 2011, 1-6

20. G.M. Pereira da Silva e Costa, I. Martins Carvalho, A.I. Rodrigues Castro, N.C. Ribeiro Lages, C.M. Machado Correia, „Postoperative analgesia for hip arthroplasty: comparison of continuous lumbar plexus block and epidural analgesia", Revista Dor. Sao Paulo, 2016, 17(1), 2-7 